\title{
DETERMINANTES SOCIAIS DE SAÚDE: características da comunidade e trabalho das enfermeiras na saúde da família ${ }^{a}$
}

\author{
Cynthia Fontella SANT 'AN NA ', M arta Regina CE ZAR-VAZ', L eticia Silveira CA R D OSO',

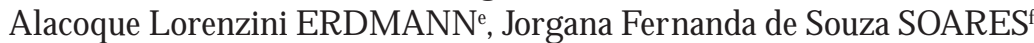

\section{RESUMO}

Este estudo objetivou identificar os Determinantes Sociais de Saúde que despontam nos depoimentos das enfermeiras, ao caracterizarem a comunidade, anal isando sua relação com o trabal ho desenvolvido. T rata-se de um estudo exploratório-descritivo com análise qualitativa nas categorias teóricas dos determinantes. U tilizou-se entrevista semiestruturada gravada com consentimento das 65 enfermeiras da E stratégia Saúde da F amília, pertencentes à 3a Coordenadoria Regional de Saúde do R io G rande do Sul. E videnciou-se a inter e intrarrelação nos fatores determinantes da saúde, obtendo 104 citações para as características anatomofisiológicas dos indivíduos/ comunidade correspondentes aos determinantes proximais e em associação predominantemente ao trabal ho desenvolvido pelas enfermeiras. Para os determinantes intermediários houve 27 citações e, para os distais, 166, com predominante referência à local ização territorial das comunidades em áreas rurais e periféricas. As enfer meiras relataram uma estreita relação entre as características proximais e o trabal ho por elas desenvolvido, bem como visualizam a relação com os demais deter minantes na relação com o processo de adoecimento.

D escritores: E nfermagem em saúde pública. Programa Saúde da Família. D esigualdades em saúde.

\section{RESUMEN}

E ste estudio tuvo el objetivo de identificar los D eterminantes Social es de Sal ud que se destacan en las declaraciones de las enfermeras, al caracterizar la comunidad, analizando su relación con el trabajo desar rollado. Setrata de un estudio exploratorio- descriptivo con análisis cualitativo en las categorías teóricas de los determinantes. F ue utilizada la entrevista semiestructurada grabada con el consentimiento de las 65 enfermeras de la Salud de la Familia, pertenecientes a la 3a Coordinación R egional de Salud del Rio G rande do Sul, B rasil. F ue evidenciado la inter y la intrarrelación en los factores determinantes de la salud, obteniendo 104 citaciones para las características anatomofisiológicas de los individuos/ comunidad cor respondientes a los determinantes proximales y en asociación predominantementeal trabajo desar rollado por las enfermeras. Para los determinantes intermediarios hubo 27 citaciones y, para los distales, 166, con predominante referencia a la localización ter ritorial de las comunidades en áreas rurales y periféricas. L as enfermeras relataron una estrecha relación entre las características proximales y el trabajo por ellas desarrollado, además de como visualizan la relación con los demás determinantes en la relación con el proceso de enfermarse.

Descriptores: E nfermería en salud pública. P rograma de salud familiar. D esigualdades en la salud.

T ítulo: D eterminantes sociales de salud: características de la comunidad y trabajo de las enfermeras en la salud de la familia.

\section{ABST RACT}

T hepur pose of this study is to identify the Social D eter minants of $H$ eal th Carewhich emergein nurses' statements as they characterizethe community, analyzing its relation to the work carried out by them. It is an exploratory and descriptive study containing a qualitative analysis in the theor etical categories of the determinants. We used a semi-structured inter view, recorded with the permission of the 65 nurses of the $\mathrm{Family} \mathrm{H}$ ealth Care, members of the $3 \mathrm{rd}$ R egional $\mathrm{H}$ ealth Care Coordination of R io $\mathrm{G}$ rande do Sul, B razil. It has been show $n$ theinter-and intra-relation in theheal th determinant factors, obtaining 104 citations for the anatomo-physiological features of the cor responding individuals/ community to the proximal correspondents and in association, mainly, to the work car ried out by the nurses. F or intermediate determinants there were 27 citations and, for distals, 166, with predominant reference to the territorial localization of communities in rural areas and peripheries. T he nurses have stated a narrow relation betw een the proximal features and the w ork car ried out by them, as w ell as the connection with other determinants in the relation with the process of getting sick.

Descriptors: P ublic health nursing. F amily health program. H ealth inequalities.

T itle: Social determinants of health: community features and nurse w ork in family health care.

a Artigo originado da dissertação de M estrado apresentada em 2009 ao Programa de Pós-Graduação em Enfermagem (PPGEnf) da Universidade Federal do Rio Grande (FURG).

${ }^{b}$ D outoranda do PPGEnf da FURG, Bolsista da Comissão de A perfeicoamento de Pessoal de Nível Superior (CAPES), Integrante do L aboratório de Estudos de Processos Socioambientais e Produção Coletiva de Saúde (LAM SA), Rio Grande, Rio Grande do Sul, Brasil.

'D outora em Filosofia da Enfermagem, Professora Adjunta da Escola de Enfermagem da FURG, Líder do LAM SA, Rio Grande, Rio Grande do Sul, Brasil.

d M estranda do Programa de Pós-Graduação em Ciência da Saúde da FU RG, Bolsista CAPES, Integrante do LAM SA, Rio G rande, Rio Grande do Sul, Brasil.

e D outora em Filosofia da Enfermagem, Professora T itular do Departamento de Enfermagem e do Programa de Pós-Graduação em Enfermagem da U niversidade Federal de Santa Catarina (U F SC), Pesquisadora 1A do Consel ho N acional de D esenvolvimento Científico e Tecnológico (CN Pq), F lorianópolis, Santa Catarina, Brasil.

f D outoranda em Saúde Pública pelo Prog rama de Pós-G raduação em Saúde Coletiva da U niversidade Federal da Bahia (U F BA), Bolsista CN Pq, Integrante do LAM SA da FURG e do Programa Integrado em Saúde A mbiental e do T rabal hador da UF BA, Salvador, Bahia, Brasil. 


\section{INT RODUÇÃO}

Os D eter minantes Sociais da Saúde (DSS) correspondem a um conjunto de fatores que caracteriza as particularidades dos indivíduos e também refl ete sua inserção em um tempo-espaço. Os determinantes referidos constituem uma redecomplexa de fatores que se inter-relacionam e condicionam o processo saúde-doença na especificidade do indivíduo e na abrangência do modo de vida coletivo.

As características de deter minada comunidade correspondem a uma diversidade e a uma complexidade de fatores que pertencem às condições de vida e saúde local e com elas se relacionam(1). $\mathrm{N}$ esse espectro, a relação entre saúde e doença é dinâmica e constante, compreendendo a capacidade nor mativa para a vida que o indivíduo possui, ou seja, pela sua capacidade de estabelecer novas normas, mesmo que orgânicas, frente às circunstâncias apresentadas e considerando-se, assim, normal, percebendo o surgimento da doença como compatível com a vida ${ }^{(2)}$.

E $m$ uma comunidade, as características anatomofisiológicas se constituem nas particularidades dos indivíduos, que representam parcela da comunidade, os DSS proximais.

Outra parcela atrela-se aos aspectos comportamentais de diferentes grupos, que pode formar redes sociais de apoio e complemento às questões de vida e saúde, os DSS intermediários. E ain$\mathrm{da}$, considera-se as características mais externas aos indivíduos e grupos, os fatores sócio-ambientais, como DSS distais $s^{(3)}$.

A comunidade, na condição de contexto sócio-ambiental, condiciona e determina a saúde e 0 adoecimento, na relação entre os indivíduos e os sistemas ambientais em que vivem, interagindo com seres vivos e não vivos ${ }^{(4)}$. E ssa inter ação desenvolve efeitos de ação e reação, que interferem nos estados de vida em comunidade, bem como em situações de construção do próprio ambiente físicosocial, produzindo meios (in)sustentáveis à sobrevivência e à preser vação(1).

E $m$ tal conjuntura, o processo detrabalho com os el ementos/ fatores deter minantes sociais de saúde - ao conhecer e atuar junto às condições de vida e saúde de indivíduos e famílias - no âmbito da E stratégia Saúde da Família (ESF) é abordado pelo M inistério da Saúde como estratégia de reestruturação da A tenção Básica, que desenvolve a me- diação das ações estatais para melhorar as condições de vida e saúde, a partir da ação de uma equipe multiprofissional(1). Essa força de trabalho impulsiona ações promotoras a grupos com fatores de risco comportamentais e ambientais, visando adequar hábitos/ estilos de vida saudáveis, minimizando 0 aparecimento de agravos ${ }^{(5)}$.

N este âmbito do grupo multiprofissional, salienta-se o trabalho das enfermeiras como agentes propulsores das ações interativas e integrativas na relação com os difer entes grupos da comunidade. Permite-se, assim, caracterizá-las como articuladoras do trabal ho multiprofissional ao inter virem nas várias dimensões da saúde e do adoecimento dos indivíduos, família e grupo, no contexto sócio-ambiental comunitário.

No desenvolvimento do trabal ho, a comunidade, quando estimulada a participar ativamente na atenção à saúde, desenvolve a corresponsabilização com os trabalhadores da equipe, permitindo 0 desenvolvimento de um trabal ho com maior abrangência frente à deter minação social da saúde.

N esse contexto, a atuação dos trabal hadores da ESF necessita ir além dos cuidados anatomofisiológicos nos indivíduos, buscando ações de prevenção da doença e promoção da saúde na comunidade, compreendendo os aspectos sócio-ambientais. Sendo assim, o presente estudo emerge do anseio da seguinte questão norteadora: Como as enfermeiras caracterizam seu objeto coletivo de trabal ho, a comunidade, de acordo com os DSS?

N essa direção, objetivou-se identificar os D eter minantes Sociais de Saúde que despontam nos depoimentos das enfermeiras ao caracterizarem a comunidade, anal isando a relação com o seu trabaIho.

\section{MÉTODO}

Estudo com abordagem qualitativa temáti$c a^{(6)}$ - a aproximação das características das comunidades atendidas na E stratégia Saúde da Família aos D eter minantes Sociais de Saúde - na tentativa de compreender uma problemática sob a ótica dos sujeitos envolvidos, as enfer meir as, para análise do objeto de investigação em determinado tempo histórico sócio-ambiental, pois o foco é para a apreensão da enfermeira sobre o seu objeto/ sujeito coletivo da ação - a comunidade que vive em um espaço delimitado e socializado cultural e economicamente. 
0 presenteestudo originou-se de uma dissertação em enfermagem ${ }^{(7)}$, a qual utilizou o banco de dados de um macroprojetog que teve como cenário de estudo a rede daESF da T erceira Coordenadoria Regional de Saúde (3a CRS), no extremo sul do Rio Grande do Sul, Brasil. A população da pesquisa constituiu-se de 65 enfermeiras que compõem a totalidade de profissionais da categoria atuantes nas 65 equipes da ESF pertencentes à 3 a CRS, no período da coleta dos dados, realizada entre janeiro a julho de 2006. Destaca-se que foi utilizado como critério de seleção das equipes a sua constituição até o período de 2004, data em que o projeto da pesquisa foi aprovado.

0 instrumento foi testado por meio do estudo-piloto, junto a uma equipe da E SF não pertencente ao grupo sel ecionado para a amostra. A s entrevistas semiestruturadas gravadas foram norteadas pela questão dirigida às enfer meiras: Expõe as características da comunidade que compõem 0 ter ritório adscrito à utilização dos serviços, nesta U nidade Saúde da Família. Este processo foi previamente projetado com o suporte dos DSS como base teórica, para sustentar a base empírica construída a partir dos depoimentos das enfermeiras sobre as condições de vida e saúde da comunidade.

A análise dos dados foi delineada pelo suporte teórico dos DSS, para possibilitar a aproximação da base empírica do conteúdo dos depoimentos das enfermeiras. Este processo permitiu a exploração do material obtido, em acordo à aplicação aos el ementos dos DSS e princípios da ESF.

A ssim, a apresentação e análise dos dados estão divididas didaticamente nas categorias teóricas, de acordo com os DSS, em: Determinantes Proximais, representados pelas categorias operacionais relacionadas ao indivíduo, como idade, sexo e fatores hereditários; D eterminantes Intermediários, que compreendem as categorias operacionais de estilo de vida e redes sociais, comunitárias e saúde; e os D eterminantes Distais, que abran-

\footnotetext{
"I ntitulado "T rabal ho em saúde e o contexto tecnológico da Política de A tenção à Saúde da Família - uma abordagem socioambiental da produção coletiva de saúde" e inserido no edital M S/CNPq/ FAPERGS no 004/ 2007 - do Programa Pesquisa para o SU S: gestão compartilhada em saúde - processo no 0415374. Objetivou analisar as mudanças atuais no modelo de atenção básica à saúde, na organização do Sistema Ú nico de Saúde (SU S), o qual intensifica a proposta de ações abrangendo os eixos transversais da universalidade, integralidade e equidade, por meio do Programa Saúde da Família (PSF).
}

gem as condições de vida e trabal ho (escolaridade, ambiente de trabalho, fonte de renda, saneamento básico, cultura, habitação e serviços sociais e de saúde) e as condições sócio-econômicas, culturais e ambientais gerais.

Quanto aos aspectos éticos, obteve-se aprovação do Comitê de Ética em Pesquisa da Área de Saúde da U niver sidade F ederal do Rio G rande (CE PAS), sob parecer no 02/ 2004. A ssegurando a observação das normas e diretrizes que regulamentam a pesquisa com seres humanos, estabelecidas pela Resolução196/ 96 do Conselho Nacional de Saúde ${ }^{(8)}$, obteve-se expresso por escrito em duas vias o consentimento de todos os entrevistados, após serem informados acerca do direito à desistência da participação sem qualquer ônus e à preservação do anonimato da identidade pessoal, dos locais de trabal ho e dos municípios.

\section{RESULT ADOS}

$D$ a total idade das 65 enfermeiras entrevistadas, 59 são do sexo feminino e 6 do masculino, com média de idade de 37,60 , variando entre 24 e 55 anos. 0 tempo de trabalho na ESF foi de 25,93 meses, variando entre 1 e 60 meses de atuação. D estes trabalhadores, 50 possuem Especialização em Saúde da F amília e 15, Especialização em Saúde Pública e Saúde Coletiva. A cerca da formação pós-graduada stricto sensu, 4 enfermeiras possuem M estrado.

Os resultados apresentados a partir das categorias teóricas - determinantes proximais, determinantes intermediários e determinantes distais são descritos com apresentação do número de refer ências feitas a cada categoria oper acional, representada entre parênteses, visando demonstrar a representação quantitativa para consubstanciar 0 qual itativo da caracterização das enfer meiras acerca das características da comunidade.

\section{D eterminantes Proximais e características anatomofisiológicas}

$\mathrm{N}$ a categoria teórica dos DSS proximais, houve 104 citações das entrevistadas referentes à faixa etária (45), ao sexo (31) e aos fatores hereditários (28), no que concer ne às car acterísticas da comunidade.

$\mathrm{Na}$ categoria operacional referente à faixaetária foi possível visualizar predominantemente 
nas falas das enfermeiras como característica proximal o idoso (25), relacionando-o com DSS distais representados pela fonte de renda, aposentadoria e melhores condições de moradia. As demais características etárias (20) foram associadas à escolaridade, para o cadastramento no bolsa-escola, e às ações desenvolvidas pela trabalhadora, no atendimento à puericultura, pré-natal, vacinação e planejamento familiar, classificadas nos DSS distais e inter mediários, respectivamente.

No que se refere ao sexo, foi descrito (25) para a diferença entre homens e mulheres, relacionada às car acterísticas distais correspondente ao tipo de emprego extra ou intradomiciliar, o último ocupado com o cuidado dos filhos; nível educacional baixo em função do trabal ho precoce; e uso de drogas, que acar retam conseqüências distintas para o homem e a mulher, desenvolvendo o alcoolismo e a depressão, respectivamente.

E $m$ relação aos fatores hereditários, foram relacionadas características que identificam patologias crônico-degenerativas (19), como a hipertensão e diabetes, depressão (8) e obesidade (1), relacionadas a outros DSS proximais, como a faixaetária (com ênfase na pessoa idosa), com DSS inter mediário, como o estilo de vida, e ainda com DSS distal, como a cultura referente aos hábitos al imentares, o sedentarismo e a ausência de atividades de lazer.

\section{D eterminantes Intermediários e interações condicionantes da vida e da saúde}

De acordo com a percepção das enfermeiras, a categoria teórica em questão obteve 27 citações, relacionadas ao estilo de vida dos indivíduos (17) e às redes sociais, comunitárias e saúde (10).

A categoria operacional estilo de vida dos indivíduos visualizou-se como predominante a referência ao uso de drogas (10), ao referir alguns DSS distais de local ização territorial ecultural, devidos à ingesta diária de bebida alcoólica, na área rural, e ao uso de substâncias ilegais como uma das causas da violência na periferia. A presenta-se ainda o comportamento pouco reivindicador associado à característica distal da cultura no território rural (6), e a atribuição de responsabilidades do cuidado entre irmãos de diferentes idades associado ao DSS distal da área periférica (1) e ainda a cultura da comunidade.
E $m$ relação às redes sociais, comunitárias e saúde, predominaram no relato das enfermeiras os espaços de lazer (8), apontados por sua ausência; e assim, utilização do serviço da unidade de saúde, a partici pação em grupos religiosos, no consel ho local de saúde e em grupo de saúde mental como meios de social ização; relacionada ao comportamento suicida (1), por sua vez, associado ao desemprego e à consequente fal ta de alimentação, ambos DSS intermediários.

\section{D eterminantes D istais e características sócio-ambientais}

N esta categoria teórica, os entrevistados fizeram 168 referências aos aspectos de condições de vida e trabalho e de condições sócio-econômicas, culturais e ambientais gerais, identificadas na educação (40), ambiente de trabalho (32), fonte de renda (29), saneamento básico (27), habitação (13), serviços sociais de saúde (13) e cultura (14).

E m relação à categoria operacional educação, 0 analfabetismo e o baixo nível escolar, identificado no 10 grau incompleto (33), foram registrados como sendo a principal característica da comunidade, relacionada aos seguintes DSS distais: Iocalização periférica ou rural, inserção precoce no mercado de trabalho, carência na alimentação e evasão escolar durante a gravidez.

Para o ambiente de trabalho foi referido como característica principal o tipo de trabal ho (25), na relação com DSS de localização, identificando na área rural o trabal ho ag rícola e ag ropecuário, e estes, por sua vez, com os DSS proximais de patologias, devido à exposição a toxinas e ao trabalho excessivo. $\mathrm{N}$ a área periférica (7), 0 ambiente de trabalho foi associado a DSS distais, como o trabal ho autônomo, sem carteira assinada.

$\mathrm{E} m$ relação à categoria operacional da fonte de renda, as enfer meiras caracterizam a comunidade pel o baixo nível salarial (15), ao referirem relação com outros DSS distais, como o trabal ho sem renda fixa e a cultura, devido ao el evado número de filhos: 11 a 12 por família. Foram apontados 0 desemprego (9) em relação aos intervalos da safra agrícola rural, característica distal, e a utilização de benefícios do governo, característica intermediária.

E m relação ao saneamento básico foram identificadas a carência e/ ou ausência de estrutura 
para esgoto (11), água (9), luz (5) e coleta de lixo (2), relacionados à car acterística distal de local ização territorial, referindo alagamentos de ruas, na área periférica, e uso de poço artesiano e despejo de esgoto nos rios, na ár ea rural.

Os aspectos de habitação foram caracterizados pelas condições de saúde (8), relacionadas às precárias estruturas físicas e tipo de material de construção (papelão, latas), co-habitação com animais, tamanho da residência, ausência de assoal ho, precárias condições de higiene e ausência de equipamentos domésticos.

E $m$ relação aos serviços sociais de saúde foi referida a acessibilidade dos clientes à unidade de ESF (9), identificando as atividades desenvolvidas, tais como grupos de saúde, vacinação e atendimento à demanda, associados a DSS distais em razão de 0 horário de trabalho coincidir com o de funcionamento da unidade ESF, da dificul dade pela distância territorial bem como da dificuldade financeira para tal deslocamento. $\mathrm{E}$ ainda foi identificado 0 perfil reivindicador (4) da comunidade, DSS intermediário, para solicitação de maior número de consultas.

Referente à cultura, foi mencionada a etnia al emã (13), identificando a área rural, característica distal, hábitos alimentares e doenças crônicas; e a dificuldade de comunicação, que recai no uso do dialeto pomerano pela comunidade. $E$ na periferia (1), foi citada a representação da mulher na sociedade, de acordo com o maior número de filhos.

\section{DISCUSSÃO}

A estruturação dos DSS expressa a mediação existente entre as diferentes categorias teóricas nas intra e inter-relações com os fatores oper acionais ${ }^{(9)}$ que, nessa dinâmica interativa do indivíduo e da coletividade aos fatores sócio-ambientais, originase a necessidade de uma assistência integ ral à saú$\mathrm{de}^{(3)}$.

0 estabelecimento da relação das características da comunidade relatadas pelas enfermeiras com os DSS evidencia o idoso pela estabilidade financeira que a aposentadoria pode significar, sendo por vezes a única fonte de renda familiar, e com possibilidade de proporcionar melhoria quantitativa e qual itativamente às condições de vida(10). Esta relação torna clara a inter rel ação entre os diferentes DSS, pois ao descrever características de uma determinada faixa etária, DSS proximal, a traba-
Ihadora faz referência à condição sócio-econômica deste, o trabalho, correspondente a um dos DSS distais.

Este mesmo sentido é identificado quando é feito referência à criança na rel ação com a escolaridade que pode representar o suporte financeiro proporcionado pelo governo, que tem o objetivo de incentivar a educação por meio de benefícios. E stes, nas áreas de carência sócio-econômica, podem significar a existência de uma criança como provedora financeira de uma família(11).

$\mathrm{N}$ este espectro, as enfer meiras caracterizam o próprio objeto coletivo de intervenção, a comunidade, a partir do desenvolvimento de seu trabaIho, direcionado às características correspondentes à faixa-etária e fatores hereditários pautado nas prioridades previstas no Pacto pela Saúde ${ }^{(5)}$, na realização de ações individuais e coletivas de responsabilidade criativa e organizativa das enfermeiras.

As enfermeiras enfatizam a relação entre 0 trabal ho masculino efeminino, considerando o primeiro como provedor financeiro de uma família, 0 que pode representar a evasão escolar por parte dos adolescentes do sexo masculino, ligados a aspectos culturais e fatores de local ização ter ritorial. D esta forma, visualiza-se a direta relação de um DSS proximal, o sexo, com o trabal ho dos indivíduos homens inter ligado à cultura, cor respondentes aos DSS distais; bem como, a intra-relação nos el ementos dos DSS distais, pois a cultura de o homem ser o provedor do sustento familiar pode interferir no processo de escolarização deste indivíduo, que corresponde também a um deter minante distal.

$\mathrm{N}$ a diferença entre sexos, percebe-se a estreita relação com o consumo de álcool, associado ao comportamento de risco, permeada às diferenças regionais e 0 tipo de bebida consumida. Pode-se constatar também a prevalência do consumo de álcool por homens, e nas mulheres, a prevalência de desordens no consumo de drogas controladas ${ }^{(3)}$. A ssim, o DSS proximal, sexo, é identificado na reIação com um DSS inter mediário, estilo de vida, 0 qual pode representar um fator de vulnerabilidade à saúde do indivíduo - correspondente a um DSS proximal, e interferir na inserção/ relação com redes sociais, comunitárias e saúde - um DSS intermediário.

V isualiza-se que o foco de abordagem aos fatores hereditários encontra-se direcionado à prevenção e controle da hipertensão e diabetes, defi- 
nidas como prioridades na atenção à saúde do adulto. Assim, compreendendo a saúde como uma questão de bons hábitos ${ }^{(12)}$, as enfermeiras na ESF desenvolvem a atenção aos indivíduos acometidos de doenças crônico-degenerativas, em atividades educativas e informativas individuais ou coletivas, incluindo conteúdos relativos a patologia, suas implicações e riscos. As referidas atividades têm em vista a perspectiva de mudanças de hábitos comportamentais e al imentares, incentivo à atividade física, entre outras atitudes possíveis para produzir a adequação dos indivíduos a hábitos mais saudáveis.

Ao analisar a descrição das enfer meiras acerca das características anatomofisiológicas da comunidade, DSS proximal, é identificado a inter rel ação que estas trabal hador as fazem com os demais DSS, apontando assim que, ainda que exista divisão teórica destes deter minantes, os fatores se conectam e interagem constantemente na vida do sujeito indivíduo e coletivo.

T al inter ação entre os DSS também pode ser visualizado em relação ao modo condicionante do viver, no qual as enfermeiras percebem a relação entre o estilo de vida e o meio de inserção dos indivíduos, que pode ter como fator diferenciador a cultura de determinada local ização ter ritorial. N essa direção, destaca-se a área rural e o uso de drogas associados às questões culturais e patologias crônico-degenerativas.

$\mathrm{Na}$ área periférica, o uso de drogas, aqui representadas prioritariamente pelas ilegais, imbrica-se com demais fatores relacionados às redes sociais e comunitárias, como o desemprego. E ste pode ser um elemento desencadeador, devido à carência nas condições de sobrevivência; um estímulo à violência, inclusive contra si mesmo, representada no comportamento suicida: em consequência da marginal ização e da exclusão social. 0 que produz, em contrapartida, 0 aumento abusivo do uso de drogas legais e il egais ${ }^{(13)}$. E sta associação de fatores representa a dinâmica relação dos determinantes, no qual um DSS inter mediário, estilo de vida, reflete inúmeras conseqüências às condições de vida e saúde, e assim interfere nos demais fatores determinantes.

Q uanto às redes sociais e comunitárias, é identificado que a localização territorial das unidades de comunidades adstritas à ESF car ece de espaços estruturados para o lazer. E ssas redes de apoio social podem representar um potencializador à redu- ção de transtor nos mentais, ainda que igualmente relacionado à idade, escolaridade e participação no mercado de trabalho, pois promove inter-relações, produzindo autoconfiança e poder de enfrentamento dos problemas no cotidiano dos indivíduos $^{(3)}$.

Para o enfrentamento de tais problemas, os trabal hadores devem consider ar que a vida é polaridade e, por isso, uma posição inconsciente de valor, sendo a restauração da doença uma atividade normativa, conforme o contexto, dentro de um dinamismo estabelecido pelo ambiente, situado no tempo e no espaço(2).

$\mathrm{N}$ a dinâmica existente da condição de saúde e adoecimento apresentada, enfatiza-se o crescimento das desigual dades sócio-econômicas e seu efeito em cadeia ${ }^{(14)}$. O u seja, o estado de bem-estar é representado por elementos anteriores e consecutivos, visto que 0 acesso à educação - DSS distal - se relaciona às possibilidades de acesso no mercado de trabal ho - outro DSS distal - produzindo a aquisição de renda ou ausência da mesma. Essa última, é importante frisar, dificulta ou impossibilita aquisições de materiais e produtos necessários à mel horia ou manutenção das condições de vida, alimentação e higiene ${ }^{(15)}$ - DSS intermediário. T orna-se oportuno, ainda, salientar a estreita relação entre o nível da escolaridade ea taxa de fecundidade, que ocorre em medida inversamente proporcional(3).

0 ambiente como um fator condicionante ao trabal ho é referido pela negligência de al guns cuidados que pode representar/ desenvolver um agravo à saúde, como na exposição a agrotóxicos visual izada na área rural, trabal ho que se desenvolve de ger ação em geração ${ }^{(16)}$. N esse sentido, a enfermeira e a sua equipe de saúde têm a possibilidade de desenvolver ações acerca da saúde do trabalhador e da prática do autocuidado.

I dentifica-se ainda que as enfermeiras caracterizam sua comunidade quanto às condições de moradia, relacionando-as à localização territorial. A periférica é inferida como a de menor acesso aos serviços de saneamento básico - DSS distal - na qual há também pouca procura pelos serviços de saúde ${ }^{(17)}$, DSS intermdiário. Dessa forma, 0 ambiente natural condiciona incisivamente o viver dos indivíduos ${ }^{(18,19)}$, seja impondo limites para locomoção, tipo de produção ou socialização, entre outros aspectos.

A partir daí, a saúde e seus determinantes necessitam ser pensados na dimensão social, cul- 
tural e econômica, que se manifesta no ambiente onde o indivíduo e sua col etividade se inserem. Pois, possibilita identificar melhor sel eção, adequação e planejamento das ações a serem desenvolvidas, objetivando a integralidade e a univer salidade da atenção, como princípio de justiça social ${ }^{(20,21)}$.

Entende-se, assim, a saúde como uma estrutura de organizações que consiste no conjunto de relações de produção de componentes do ambiente, os quais se modificam e transformam as relações entre si, à medida que vão interagindo com/ no ambiente, sendo o ser humano o produto da atividade social. O u seja, um conteúdo complexo, que no nível da ciência, da saúde, é uma categoria ou conceito com suas aplicações, real izando-se estruturalmente no tempo e no espaço ${ }^{(1)}$, enfatizando a conexão das relações complexas e mútuas entre 0 sujeito e o seu ambiente.

\section{CONSIDERAÇÕES FINAIS}

F rente ao exposto, o estudo al cançou o objetivo proposto, possibilitando visualizar a relação direta existente entre ambiente e saúde, pois as condições da última podem interferir no ambiente físico, econômico e social, bem como o inverso também se torna verdadeiro.

Pode-se, assim, concluir que as enfermeiras daESF caracterizam seu objeto de intervenção por meio de aspectos condicionantes do modo de viver, trabal har e se relacionar dos clientes. T ais condicionantes atuam como um fator determinante do nível comunitário de saúde, compreendendo a dinâmica de interação dos indivíduos em coletividade, as estruturas anatomofisiológicas, relações inter pessoais e ambientais.

Assim, a percepção dos determinantes proximais descreve particularidades dos indivíduos observadas nas ações de trabal ho desenvolvido peIas enfermeiras. Elas, ao desenvolverem suas ações na direção dessas particularidades, interagem diretamente com os indivíduos nas diversas prioridades de cada período do ciclo vital, as quais exigem manutenção do processo saúde-doença, inserindo-os nos serviços de saúde como objeto de intervenção. Destaca-se que, no trabalho em ESF, esses indivíduos também são visualizados em sua coletividade e, assim, o desenvolvimento da atenção básica excede os aspectos da doença ${ }^{(1)}$.

Consecutivamente, as enfer meiras relacionam o processo saúde-doença aos fatores que mediam as relações interpessoais, uma vez que elas podem definir hábitos e comportamentos. Por outro lado, os el ementos mencionados, em um coletivo, podem produzir uma rede cogestora dos serviços de saúde, entendida evisualizada como um ambiente promotor da cidadania, ao desenvolver ações solidárias às necessidades locais ${ }^{(1)}$.

E ssas necessidades sócio-ambientais são consideradas pelas enfermeiras nas diferentes inserções dos indivíduos no mercado de trabal ho, ou às margens deste, como fator determinante ao modo e às condições de vida, refletindo nas ações e costumes dos mesmos. Assim, eles consideram, além do aspecto da remuneração e do poder aquisitivo, os fatores de infraestrutura do ambiente para consumo coletivo, incluindo serviços de saúde, saneamento básico, acesso à educação, alimentação, lazer, segurança, transporte, entre outros ${ }^{(8)}$.

$\mathrm{N}$ essa complexidade de fatores que inter agem no ambiente comunitário, é possível perceber duas relações principais: de direta relação dos DSS proximais às ações de saúde desenvolvidas no trabal ho das enfermeiras; e na correlação com os serviços sociais e de saúde, DSS intermediários e distais, que enfatizam o acesso dos indivíduos ao processo de trabalho em ESF.

Portanto, percebe-se que ao conhecer os elementos/ fatores determinantes às condições de vida e saúde dos indivíduos e da comunidade torna-se possível proporcionar ações mais adequadas, ou seja, embasam a organização do trabal ho de cuidado das enfermeiras visando proporcionar melhores condições à qualidade de vida, bem como visualizar e intervir para transfor mação de deter minados DSS que representam aspectos negativos à saúde da população.

E assim, destaca-se o trabal ho desenvolvido pelas enfermeiras na E stratégia Saúde da Família, o qual pode ser considerado uma parceria de sucesso para o desenvolvimento de uma atenção integral, que inclui os princípios da atenção básica ambiental como instrumentos na produção do processo saúde/ doença.

\section{REFERÊ NCIAS}

1 Cezar-Vaz M R, Soares M CF, M artins SR, Sena J, Santos $L R, R$ ubira $L T$, et al. Saber ambiental: instrumento interdisciplinar para a produção de saúde. T exto Contexto Enferm. 2005;14(3):391-7.

2 Canguilhem G. 0 normal e o patológico. Rio de Janeiro: Forense U niversitária; 1966. 
3 Comissão Nacional sobre Determinantes Sociais da Saúde. Relatório final da Comissão Nacional sobre Determinantes Sociais da Saúde [I nternet]. Rio de Janeiro; 2008 [ citado 2008 dez 06] . Disponível em: http:/ / www.cndss.fiocruz.br/ pdf/ home/ relatorio.pdf.

4 Sistemas e ecossistemas: definições. In: Frontier S. Os ecossistemas. Lisboa: I nstituto Piaget; 2001. p. 13-30.

5 M inistério da Saúde (BR ). Pacto pela saúde. Portaria no 399/GM , de 22 de fevereiro de 2006: divulga 0 Pacto pela Saúde 2006 - Consolidação do SU S e aprova as diretrizes operacionais do referido pacto [ Inter net] . Brasília (D F ); 2006 [ citado 2009 abr 04] . Disponível em: http:/ / bvsms.saude.gov.br/bvs/ publicacoes/ prtG M 399_20060222.pdf.

6 M inayo M CS. 0 desafio do conhecimento: pesquisa qualitativa em saúde. 4ạ ed. São Paulo: HucitecAbrasco; 2004.

7 Sant'A nna CF. Interação enfermeira-comunidade na E stratégia Saúde da F amília: um estudo das características do sujeito e da finalidade [ dissertação] . Rio Grande: U niversidade Federal do Rio Grande; 2009.

8 M inistério da Saúde (BR), Conselho N acional de Saúde. Resolução 196, de 10 de outubro de 1996: diretrizes e normas regulamentadoras de pesquisa envolvendo seres humanos. Brasília (D F ); 1996.

9 Buss PM , Filho AP. A saúde e seus determinantes sociais. Physis. 2007;17(1):77-93.

10 Cupertino A P F B, Rosa F H M , R ibeiro PCC. D efi-nição de envel hecimento saudável na perspectiva de indivíduos idosos. Psicol Reflex Crít. 2007;20(1): 81-6.

11 M inistério Público (RS). Projeto de L ei $n .4 .975$, de $1^{\circ}$ de agosto de 2001: al tera os arts. $2^{\circ}, 4^{\circ}$ e $5^{\circ}$ da L ei $n^{\circ} 10.219$, de 11 de abril de 2001 e dá outras providências [ I nternet] . Porto A legre; 2001 [ citado 2009 ago 22] . Disponível em: http:/ / www.mp.rs.gov.br/ infancia/ legislacao/ id2578.htm.

12 H eringer A, Ferreira VA, A cioli S, Barros A L S. Práticas educativas desenvolvidas por enfermeiros do

\section{Endereço da autora / Dirección del autor / Author's address:}

Cynthia Fontella Sant'A nna

Rua Domingos de A Imeida, 1541, ap. 302, Centro

97500-002, U ruguaiana, RS

E - mail: cynthiafs_enf@yahoo.com.br
Programa Saúde da Família no Rio de Janeiro. Rev G aúcha E nferm. 2007;28(4):542-8.

13 G uimarães J M X, Vasconcel os E E, Cunha RS, M elo RD, Pinho LF. Estudo epidemiológico da violência por arma branca no município de Porto Grande, A mapá. Ciênc Saúde Colet. 2005;10(2):441-51.

14 Santos D L , G erhardt T E . D esigualdades sociais e saúde no Brasil: produção científica no contexto do Sistema Ú nico de Saúde. Rev G aúcha Enferm. 2008; 29(1):129-36.

15 M aragno L, G oldbaum M , G ianni RJ, N ovaes H M D, César CL G. Prevalência de transtornos mentais comuns em populações atendidas pelo Programa Saúde da Família (QUALIS) no município de São Paulo, Brasil. Rev Saúde Pública. 2006;22(8):1639-48.

16 F aria N M X , F assa AG , F acchini L A . Intoxicação por agrotóxicos no Brasil: os sistemas oficiais de informação e desafios para realização de estudos epidemiológicos. Ciênc Saúde Colet. 2007;12(1):25-38.

17 Stülp VJ. E feitos dos setores econômicos e da escolaridade sobre o rendimento do trabal ho no Rio G rande do Sul. Rev E con Sociol Rural. 2006;44(1):99-117.

18 G ünther IA. E spaços de vida: aspectos da relação homem-ambiente. E stud P sicol. 2003;8(2):341-3.

19 Silva LA A, M ercês N NA, Schmidt SM S, M arcelino $\mathrm{SR}$, Pires DEP, Carraro TEL. U m olhar sócioepidemiológico sobre o viver na sociedade atual esuas implicações para a saúde humana. T exto Contexto E nferm. 2006;15(n esp):170-7.

20 L uiz OC, H eimann LS, Boaretto RC, Pacheco AG, Pessoto U C, Ibanhes LC, et al. Diferenciais intermunicipais de condições de vida e saúde: construção de um indicador composto. Rev Saúde Pública. 2009;43(1):115-22.

21 Cohen SC, Cynamon SE, K liger man D C, A ssumpção RF. H abitação saudável no P rograma Saúde da Família (PSF): uma estratégia para as políticas públicas de saúde e ambiente. Ciênc Saúde Colet. 2004;9(3): 807-13.

Recebido em: 25/ 10/2009

A provado em: 14/ 01/ 2010 